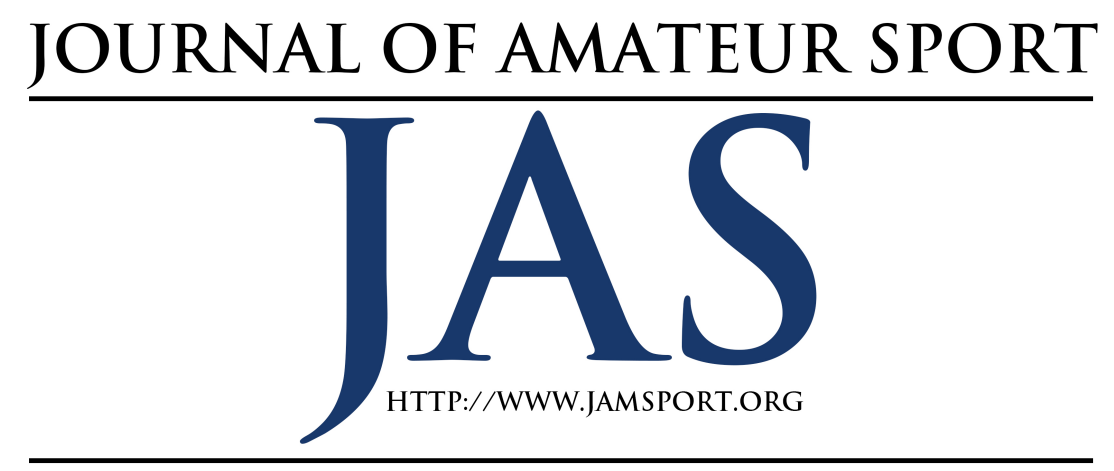

\title{
Journal Leadership and Editorial Board
}

\section{Leadership}

Co-Editor and Founder: Jordan R. Bass, University of Kansas

Co-Editor: Brian S. Gordon, University of Kansas

Associate Editor: Kyle S. Bunds, North Carolina State University

Associate Editor: Mark Vermillion, Wichita State University

Contributing Executive Council: Earle Zeigler

Production Director: Claire C. Schaeperkoetter, University of Kansas

Kwame Agyemang

Emeka Anaza

Chris Barnhill

Jonathan Casper

Cassandra Coble

Simon Darnell

Travis Dorsch

Brendan Dwyer

Travis Feezell

Rick Grieve

Marion Hambrick

Meg Hancock

Cody Havard

James Johnson

Timothy Kellison

\section{Editorial Board}

\author{
Louisiana State University \\ James Madison University \\ Louisiana State University \\ North Carolina State University \\ Indiana University \\ University of Toronto \\ Utah State University \\ Virginia Commenwealth University \\ University of the Ozarks \\ Western Kentucky University \\ University of Louisville \\ University of Louisville \\ University of Memphis \\ Ball State University \\ University of Florida
}


Shannon Kerwin

Ryan King-White

Geoffery Kohe

Leeann Lower

Marshall Magnusen

Brian McCullough

Chad McEvoy

Nicole Melton

Katie Misener

Joshua Newman

Calvin Nite

Dawn Norwood

Julie Partridge

Ted Peetz

Ann Pegoraro

Adam Pfleegor

Lamar Reams

Steve Ross

Michael Sam

Jimmy Sanderson

Chad Seifried

Clay Stoldt

Sarah Stokowski

Nefertiti Walker

Daniel Wann

Stacy Warner

Janelle Wells

Craig Williams

Masa Yoshida
Brock University

Towson University University of Worcester

Ball State University

Baylor University

Seattle University

Northern Illinois University University of Massachusetts Amherst

University of Waterloo

Florida State University

Texas Tech University

Wingate University

Southern Illinois University

Belmont University

Laurentian University

Mississippi State University

Old Dominion University

University of Concordia - St. Paul

University of Otago

Clemson University

Louisiana State University

Wichita State University

University of Arkansas

University of Massachusetts Amherst

Murray State University

East Carolina University

University of South Florida

University of Exeter

Biwako Seikei Sport College 